\title{
Description of complex flow behaviour using global dynamic modes
}

\author{
DAN S. HENNINGSON
}

Linné FLOW Centre, KTH Mechanics, Stockholm, Sweden

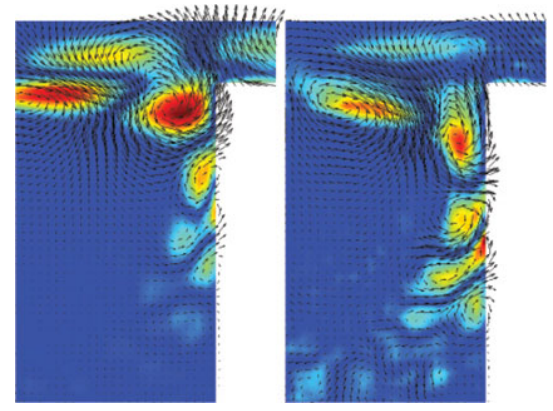

A novel method for performing spectral analysis of a fluid flow solely based on snapshot sequences from numerical simulations or experimental data is presented by Schmid (J. Fluid Mech., 2010, this issue, vol. 656, pp. 5-28). Dominant frequencies and wavenumbers are extracted together with dynamic modes which represent the associated flow structures. The mathematics underlying this decomposition is related to the Koopman operator which provides a linear representation of a nonlinear dynamical system. The procedure to calculate the spectra and dynamic modes is based on Krylov subspace methods; the dynamic modes reduce to global linear eigenmodes for linearized problems or to Fourier modes for (nonlinear) periodic problems. Schmid (2010) also generalizes the analysis to the propagation of flow variables in space which produces spatial growth rates with associated dynamic modes, and an application of the decomposition to subdomains of the flow region allows the extraction of localized stability information. For finite-amplitude flows this spectral analysis identifies relevant frequencies more effectively than global eigenvalue analysis and decouples frequency information more clearly than proper orthogonal decomposition.

\section{Introduction}

The analysis of complex flows using global modes has become commonplace among fluid dynamicists. The term 'global modes' has been used variedly to denote entities such as linear eigenmodes that depend on more than one inhomogeneous-direction (Theofilis 2003), proper-orthogonal-decomposition (POD) modes that characterize the most energetic fluid elements in the flow and balanced modes that represent flow structures that are most easily forced and measured by actuators and sensors in flow-control applications (Bagheri, Brandt \& Henningson 2009a). With the rapid advances in computer power and numerical methods, the calculation of such global modes has become feasible. For stability calculations the method of choice is a Krylov subspace technique (Edwards et al. 1994; Barkley, Blackburn \& Sherwin 2008), while for POD modes and balanced modes efficient snapshot methods have been devised (Sirovich 1987; Ilak \& Rowley 2008). However, none of these methods extracts structures associated with dominant frequencies for finite-amplitude flows, and most are difficult to apply to flow fields obtained from experiments. The novel dynamic mode decomposition (DMD) of Schmid (2010) generalizes the concept of 
global modes to these situations and can be used to describe complex flow behaviour using a reduced number of degrees of freedom.

\section{Overview}

The DMD is a technique to process a sequence of snapshots sampled in constant intervals $\Delta t$ from numerical simulations or experiments. Let us denote the $k$ th flow field of this sequence by $u_{k}$ and assume the dynamical system that generated this sequence to be in the form

$$
u_{k+1}=f\left(u_{k}\right) \text {. }
$$

For linear problems, the right-hand side of (2.1) reduces to $f\left(u_{k}\right)=A u_{k}=\mathrm{e}^{L \Delta t} u_{k}$, with $L$ representing the linearized governing equations. A spectral analysis of the linear problem then amounts to finding the global eigenmodes of $A$. The DMD method generalizes the global stability analysis to the case when the snapshot sequence is governed by a nonlinear process. Assuming a linear mapping between the snapshots, a modified Arnoldi technique (see Ruhe 1984) is used to compute a low-dimensional representation of this mapping on the basis of snapshots. Mathematically, this representation approximates the 'Koopman operator' which is at the base of the DMD. The Koopman operator $K$ is defined as (see Mezic 2005)

$$
K g\left(u_{k}\right)=g\left(f\left(u_{k}\right)\right)=g\left(u_{k+1}\right),
$$

with $g(u)$ being an observable of the flow field $u$, say, its kinetic energy. In (2.2), the linear operator $K$ replaces the nonlinear mapping $f$ of $(2.1)$; it is equivalent to the assumed linear mapping $A$ between the snapshots.

The eigenvalues $\mu_{j}$ of $K$ give information about the temporal evolution of the observable over the given time interval $\Delta t$. The associated eigenfunctions $\varphi_{j}$ of $K$ can be used in an expansion of the flow field. The complete recovery of the snapshot sequence is then an eigenfunction expansion (see Rowley et al. 2009)

$$
u_{k}=\sum_{j=1}^{\infty} K^{k} \varphi_{j} v_{j}=\sum_{j=1}^{\infty} \mu_{j}^{k} \varphi_{j} v_{j}
$$

where $\varphi_{j}$ is the amplitude, and $\mu_{j}^{k}$ and $v_{j}$ describe the temporal evolution and the spatial structure, respectively. The DMD is a numerical technique to extract the components of expansion (2.3) from a finite number of snapshots. For snapshots stemming from a linear process, the Koopman modes $v_{j}$ reduce to the linear global eigenmodes of the linear process, while for nonlinear periodic problems, they are related to Fourier modes (see Rowley et al. 2009).

Critically, spectral properties of the flow are deduced directly from the data, without reliance on an underlying model or set of equations. This particular feature introduces great flexibility. For example, the evolution in time (in the above example) can be replaced straightforwardly by an evolution in a spatial coordinate direction. By a simple reorganization of the data into a spatially arranged sequence of snapshots, the DMD will produce an inter-snapshot mapping that contains information about preferred spatial wavenumbers and associated spatial growth/decay rates. In addition, concentration on subdomains of the flow field allows the concentration on localized flow phenomena and the dissection into various co-existing or competing dynamic mechanisms. A further consequence of the data-based approach is the possibility of processing composite observables of the flow. For example, time-synchronized 
(a)

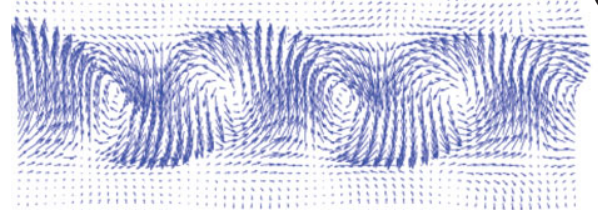

(b)

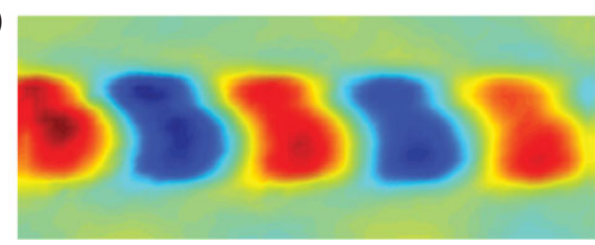

Figure 1. Dominant DMD mode extracted from time-resolved particle image velocimetry (PIV) measurements of the wake flow behind a flexible membrane, visualized by $(a)$ vector plots and $(b)$ contours of the transverse velocity component.

measurements of velocity fields by PIV and of acoustic pressure signals from a microphone array in the far field can be processed together to investigate the aeroacoustic properties of a compressible flow.

An interesting example from Schmid (2010) showing the potential of the DMD method features the spectral analysis of a flow past a U-shaped steel frame holding a flexible latex membrane. Time-resolved PIV measurements from the wake are taken in the plane of the steel frame and processed by using the DMD algorithm. In a first pass, the snapshots have been aligned in time, yielding a temporal analysis of the flow field. A distinct (non-zero) frequency could be detected corresponding to a nearly neutral dynamic mode. The corresponding dynamic mode shows large-scale vortical structures with a well-defined spatial scale and a weak amplitude decay in the streamwise direction, as shown in figure 1. A subsequent spatial DMD analysis of the same flow along the streamwise direction reproduced a preferred wavenumber and spatial growth rate decay rate of the dynamic mode. A quantitative equivalence between the spatial and temporal characteristics can be established, thus confirming that the identical spatio-temporal structure has been identified by a temporal and spatial DMD analysis (see Schmid 2010).

\section{Future}

The versatility of the DMD allows its application to a variety of evolution processes and data formats, ranging from time-resolved PIV measurements to image sequences. Fluid dynamical, acoustic, reactive, thermal and fluid-structural systems can be analysed as long as the processed data accurately capture the relevant temporal and/or spatial scales of the dynamic process. In the figure beside the title, two representative dynamic modes extracted from the experimental data of flow over an open cavity at an intermediate Reynolds number are visualized by vorticity contours and velocity vectors. These images (L. Pastur \& F. Lusseyran, 2010, private communication) demonstrate the power of this technique in very complex situations.

As a final demonstration and a further generalization of the spatial DMD framework, snapshots from experiments or numerical simulations do not have to align with a particular coordinate direction, but instead may be taken along the curved streamlines of a base flow. This is demonstrated by processing flow fields from direct numerical simulations of a jet in crossflow (Bagheri et al. 2009b) projected onto planes normal to the base-flow streamline, emanating from the jet nozzle, and arranged equispaced along its arclength (see figure $2 a$ ). For the dominant Strouhal number, a spatial instability with a distinct spatial wavenumber along the curved 

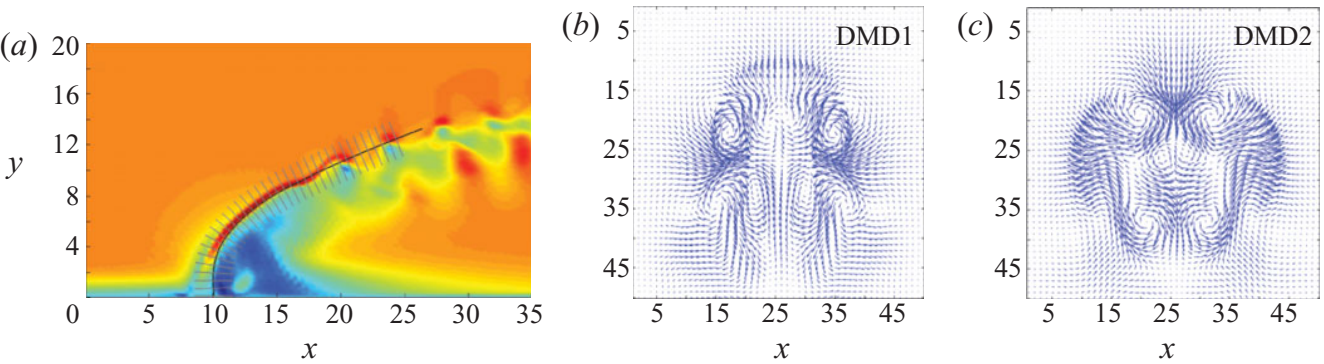

FiguRE 2. Spatial DMD along a curved streamline for a jet in crossflow. (a) Flow field snapshot visualized by the streamwise velocity component. The base-flow streamline emanating from the jet exit is shown in black; the planes normal to this streamline (onto which the velocity field is projected) are visualized in grey. $(b, c)$ Two DMD modes are in the cross-plane normal to the curved streamline (P. Schmid \& L. Bagheri, private communication 2010).

streamline could be detected. Two representative dynamic modes (figure $2 b, c$ ) show coherent vortical patterns near both flanks of the counterrotating vortex pair. These examples illustrate the potential of the DMD method for extracting useful information from experimental or numerical data sets. It is clear that there will be many others.

Special thanks to P. Schmid and S. Bagheri for the possibility to include the spatial DMD analysis of the jet in crossflow, and for their insightful comments.

\section{References}

BAgheri, S., Brandt, L. \& Henningson, D. S. $2009 a$ Input-output analysis, model reduction and control of the flat-plate boundary layer. J. Fluid Mech. 620, 263-298.

Bagheri, S., Schlatter, P., Schmid, P. J. \& Henningson, D. S. $2009 b$ Global stability of a jet in crossflow. J. Fluid Mech. 624, 33-44.

Barkley, D., Blackburn, H. M. \& Sherwin, S. J. 2008 Direct optimal growth for timesteppers. Intl J. Numer. Methods Fluids 57, 1435-1458.

Edwards, W. S., Tuckerman, L. S., Friesner, R. A. \& Sorensen, D. C. 1994 Krylov methods for the incompressible Navier-Stokes equations. J. Comp. Phys. 110, 82-102.

ILAK, M. \& RowleY, C. W. 2008 Modeling of transitional channel flow using balanced proper orthogonal decomposition. Phys. Fluids 20, 034103.

Mezic̀, I. 2005 Spectral properties of dynamical systems, model reduction and decompositions. Nonlinear Dyn. 41, 309-325.

Rowley, C. W., Mezic, I., Bagheri, S., Schlatter, P. \& Henningson, D. S. 2009 Spectral analysis of nonlinear flows. J. Fluid Mech. 641, 115-127.

RuHe, A. 1984 Rational Krylov sequence methods for eigenvalue computation. Linear Algebr. Appl. 58, 391-405.

Schmid, P. J. 2010 Dynamic mode decomposition of numerical and experimental data. J. Fluid Mech. 656, 5-28.

Sirovich, L. 1987 Turbulence and the dynamics of coherent structures. Part I. Coherent structures. Q. Appl. Math. 45, 561-571.

Theofilis, V. 2003 Advances in global linear instability analysis of nonparallel and three-dimensional flows. Prog. Aerosp. Sci. 39, 249-315. 